\title{
Editorial: Environmental Informatics for Environmental Planning and Management
}

\author{
Guest Editor: Xinghao Wang ${ }^{*}$ \\ School of Planning, University of Cincinnati, Cincinnati, OH 45221-0016
}

\begin{abstract}
Environmental information technologies have played important roles in environmental planning and management towards sustainable development strategies. One of the widely recognized features of the information process is the use of Geographic Information Systems (GIS), Geo-spatial statistics, simulation modeling, and any combination of them. Many research projects have explored the potential of using GIS to store and analyze spatial data, as well as to present data and analytical results with maps, tables, charts, or texts. Various simulation models have long been used in environmental studies, such as using surface and subsurface water models for resource and environmental management (Darbar et al., 1995; Cowen et al., 1995; Merchant, 1994; Smith and Vidmar, 1994; Warwick and Haness, 1994; Tim and Jolly, 1994). Other models simulate the interactions between land use and transport to connect economic activities in space with accessibility (Barra, 2001). In general, simulation models provide the information necessary for analysis and evaluation among planning alternatives (Putman and Chan, 2001). This special issue includes five essays that illustrate the wide spectrums of environmental information process and application. These essays share a common goal of using information technologies, which is to develop decision support systems that enable scientists, policy makers, planners and public to better understand the natural phenomena and environment-human interactions.
\end{abstract}

\section{The Theme Papers}

The first paper, by Yin et al., presents a GIS study analyzing the spatial patterns of lightning in the Western United States. The occurrence of lightning has been used to predict spatial pattern of wildfire occurrence and the possible enhancement effect of urbanization on lightning frequency. The study investigates the potential of using density surfaces generated by GIS as a tool for both visual and quantitative analyses of the spatial pattern of lightning strikes. The authors use a GIS approach to explore the potential of examining variation patterns of lightning strikes at different scales. A density surface is a raster presentation of point density distribution as

\footnotetext{
*Corresponding author: xinhao.wang@uc.edu
}

a continuous smooth surface over space. The level of the surface smoothness is a function of the scale (bandwidth, or search radius). The smaller bandwidth rendered slightly more details than the map based on the greater bandwidth. The density surface can become increasingly smoothed as the bandwidth increased and, concurrently, local details were diminished in the process. In their study, the authors address the issue that there are insufficient data on how well such surfaces can be used in a quantitative analysis of point distribution patterns. They quantitatively compare, using regression analysis, the lightning density values obtained by counting the number of lighting events in grids and the lightning density obtained by summarizing the density surfaces using the same set of grids. The study shows that a kernel bandwidth can be estimated once the spatial scale and a desirable performance level are determined. There is a threshold in the search radius or kernel bandwidth, above which a significant amount of errors would be introduced in quantitative analysis.

Tong et al. in the second paper, examines impacts of climate change on nutrient and sediment loads in a Mid-western agricultural watershed in the United States. Since the quality and quantity of water resources affect human life and activeties it is important to assess the potential hydrologic impacts of future climate change. The Soil and Water Assessment Tool (SWAT), continuous, physically based watershed-scale hydrologic model is used to predict long-term impacts on sediment, water flow, and chemical and nutrient (total nitrogen and total phosphorus) yields in large complex ungauged watersheds with varying soil, land use, and management conditions over time. The authors have analyzed five climate change scenarios. The base case scenario represents the current temperature and precipitation conditions, using averaging the daily temperature and precipitation values for the last fifty years (from 1945 to 1995). In addition, there are two wetter scenarios and two drier scenarios. Precipitation is increased by $20 \%$ for the two wetter scenarios and decreased by $20 \%$ for the two drier scenarios. Temperature increase of $4^{\circ} \mathrm{C}$ is applied in the wet and driest scenarios and a $2^{\circ} \mathrm{C}$ increase is for the wettest and dry scenarios. SWAT version 2000 has a GIS-interface with GRASS and ArcView (Jayakrishnan et al., 2005). It has already been incorporated into the USEPA's Better Assessment Science Integrating Point and Non-point Sources (BASINS) 
package through a SWAT Extension Interface. BASINS is a GIS ArcView-based multi-purpose environmental analysis system for watershed and water quality studies. The system contains much GIS data and many utilities for pre-processing (for example, watershed delineation, model input generation) and tools for assessment and post-processing (such as, displaying modeling results in graphs and tables and interpreting outputs). In SWAT, the hydrology of a watershed is simulated under two phases: the land phase and the water (or routing) phase. The land phase controls the amount of water, sediment, nutrient, and pesticide loadings to the main channel in each subwateshed. The routing phase is the movement of water, sediments, and nutrients through the channel network to the outlet of the watershed. The climate data together with all other GIS data are used as input into the SWAT hydrologic model with the BASINS interface. In the pre-processing, the study watershed is divided into 25 subwatersheds using the automatic delineation tool in BASINS. Parameters for each of the 25 delineated sub-watersheds are estimated by using the SWAT interface based on the predominant land-use or soil type described by the GIS map layers.

The simulation of the future climate conditions reveals that changes in runoff, nutrient and pollutant loads can range from $10 \%$ to $30 \%$. Among the variables, net precipitation, rather than temperature, has a more prominent impact on annual runoff. The study concludes that the climate change will have significant impacts on surface-water runoff. In many cases, these impacts are compounded by the inadequacies of our current water treatment systems to cope with climatic anomalies. Those changes will be large enough to require a significant planning response.

The study of the third paper by Mitsova-Boneva and Wang also involves the BASINS model. They examine the use of Monte Carlo simulation method together with a deterministic water quality simulation model to investigate the variability of sediment loadings from various land uses in a watershed in Ohio, USA. The study first obtains estimates for suspended sediment yield from urban, agricultural and forest land and then generates probability density and cumulative distribution functions for those estimates. The result shows not only the range of possible parameter values but also the probability with which those values are likely to occur. In a larger context, this knowledge will be useful in approaching issues of uncertainty and variability in the modeling process. Another component of the BASINS model, the Hydrologic Simulation Program - Fortran (HSPF), is applied to simulate stream flow and sediment loadings. The daily values for suspended sediment yield from various land uses generated by HSPF are then examined through stochastic modeling and probability density and cumulative distribution functions for the estimates are obtained with the Monte Carlo simulation technique. Monte Carlo simulation has been considered an appropriate technique for conducting probabilistic analyses in many environmental exposure and risk assessment studies. The study demonstrates that integrating the strengths of deterministic and probabilistic analysis in examining hydrologic and water quality responses at a watershed level would contri- bute to fuller understanding of the dynamics of the natural processes and provide planners, environmental risk assessors, researchers, and concerned citizens with knowledge on the possible range of outcomes.

Shearer and Xiang in their paper provide a comprehensive review of riparian buffer studies in the past three decades. The establishment and maintenance of riparian buffers have been an important environmental management practice in the United States since the 1970's. The literature starts to emerge with greater frequency in the 1980's, grows in depth and scope throughout the 1990's, and continues to the present date. Publications fall into three broad thematic categories that relate to the functions, performance, and policies of riparian buffers, respectively. Geospatial information technology, such as GIS, and advanced modeling, plays an integral role in the evolution of riparian buffer studies, such as visualizing the relationships among streams, land use and pollution within the riparian buffers in 1990s. In 2000s, the advancement in geospatial information technology, especially GIS, continued to contribute to buffer performance studies, such as delineating riparian buffer boundaries, measuring riparian buffer's potential for reducing water pollutants, and determining the best vegetation compositions for buffers. The literature also reports the integration of GIS, remote sensing imagery and quantitative assessment models to support decision-making in and around the riparian buffers. The use of imagery has eased the need for some field collection tasks. The authors find that the two most eminent characteristics of riparian buffer studies are the sustained advocacy for a holistic approach from authors across a vast range of disciplines, and the successful adaptation of geospatial information technology by the research community.

The paper by Fisher et al. demonstrates another approach of data analysis of environmental phenomena. The study applies type-2 fuzzy sets in geomorphometric analysis to estimate morphometric elements and the change of sand dunes based on high resolution digital elevation models. Because of the gradual change of environmental phenomena vagueness becomes an intrinsic nature of classification. A common representation of the vagueness is to use type-1 fuzzy sets. The paper points out the limitation of the type-1 fuzzy sets - a precise state about a vague object and applies type- 2 fuzzy sets to analyze the landforms and to explore the changing morphometry of a coastal sand dunefield. The change of the sand dune is evaluated as the total area of fuzzy change which is given by the sum of all memberships of the fuzzy set. The authors first estimate memberships of type-1 fuzzy sets from the instances of differently parameterised morphometric landform classifications and summarize them into type-2 fuzzy sets. They also directly examine change in the parameters of type-2 fuzzy sets, viewing the results for the different parameters as separate instances of change. The results exhibit a range of possible but small degrees of fuzzy change, which is expected in a coastal location in mid-latitudes. The study demonstrates the change analysis of type- 2 fuzzy sets can be an effective tool in presenting the environmental phenomena which are vague. 


\section{Environmental Planning and Management}

Environmental informatics can benefit environmental planning and management tremendously. The issues studied by environmental planners and managers always have strong spatial and temporal components. Proper use of information technologies can enhance the researchers and practitioners' ability for data collection, exploration, analysis, and presentation. The papers demonstrate that GIS and spatial statistics can help to increase efficiency and improve geographic precision, process and display spatial data. GIS also provides connection to remote sensing technology and prepares data for simulation modeling. The integration of GIS and simulation modeling substantially expands the temporal dimension of GIS applications and increase the use and understandability of simulation models. When the spatial analysis and simulation output are further integrated with statistical analysis, the findings can be empirically validated and generalized for broader applications. Overall, such integrations would make it possible to improve the process of environmental planning and management for evaluating different planning scenarios. In addition, the probability and fuzzy analytical approaches can contribute greatly to understand the vague and uncertain nature of the environment.

Environmental planners and managers have not yet used the information technologies as fully as possible (Klosterman, 2001). The collection of papers in this special issue is contributing to exhibit the importance of information technologies for environmental studies. While studies have shown the benefit of integration of GIS, remote sensing, simulation modeling, visualization, and statistical analysis researchers are still challenged to improve the efficiency and feasibility of integrating these information technologies for better planning and management support. With such an integrated approach, people will be able to evaluate and compare different scena- rios and their environmental quality consequences.

\section{References}

Barra, T. (2001). Integrated land use and transport modeling: The Tranus experience, in R.K. Brail and R.E. Klosterman (Eds.), Planning Support Systems: Integrating Geographic Information Systems, Models, and Visualization Tools, ESRI Press, Redlands, CA, pp. 129-156.

Cowen, D.J., Jensen, J.R., Bresnahan, P.J., Ehler, G.B., Graves, D., Huang, X., Wiesner, C. and Mackey, H.E. (1995). The design and implementation of an integrated geographic information system for environmental applications. Photogramm. Eng. Remote Sensing, 61(11), 1393-1404.

Darbar, P.M., Blood, E.R. and Choi, K.S. (1995). Modeling of urban nonpoint source pollution using a GIS in Charleston Harbor, South Carolina, in GIS/LIS '95 Annual Conference and Exposition Proceedings, Nashville, TN, pp. 235-246.

Klosterman, R.E. (2001). Planning support system: A new perspective on computer-aided planning, in R.K. Brail and P.E. Klosterman (Eds.), Planning Support Systems, ESRI Press, Redlands, CA.

Merchant, J.W. (1994). GIS-based groundwater pollution hazard assessment: A critical review of the DRASTIC model. Photogramm. Eng. Remote Sensing, 60(9), 1117-1127.

Putman, S.H. and Chan, S.L. (2001). The Metropilus planning support system: Urban models and GIS, in R.K. Brail and R. E. Klosterman (Eds.), Planning Support Systems: Integrating Geographic Information Systems, Models, and Visualization Tools, ESRI Press, Redlands, CA, pp. 99-128.

Smith, M.B. and Vidmar, A. (1994). Data set derivation for GIS-based urban hydrological modeling. Photogramm. Eng. Remote Sensing, 60(1), 67-76.

Warwick, J.J. and Haness, S.J. (1994). Efficacy of ARC/INFO GIS application to hydrologic modeling. J. Water Resour. Plan. Manage., 120(3), 366-381.

Tim, U.S. and Jolly, R. (1994). Evaluating agricultural nonpointsource pollution using integrated geographic information systems and hydrologic/water quality model. J. Environ. Qual., 23, 25-35. 